\title{
AN OPTIMIZED CONTENT-AWARE AUTHENTICATION SCHEME FOR STREAMING JPEG-2000 IMAGES OVER LOSSY NETWORKS
}

\author{
Zhishou Zhang ${ }^{1,2}$, Qibin Sun ${ }^{1}$, Susie Wee ${ }^{3}$ and Wai-Choong Wong ${ }^{1,2}$ \\ ${ }^{1}$ Institute for Infocomm Research, Singapore \\ 2 Department of ECE, National University of Singapore, Singapore \\ ${ }^{3}$ Hewlett-Packard Laboratories, Palo Alto, CA USA
}

\begin{abstract}
In this paper, we propose an optimized content-aware authentication scheme for JPEG-2000 streams over lossy networks, where a received packet is consumed only when it is both decodable and authentic. In a JPEG-2000 codestream some packets are more important than others in terms of coding dependency and visual quality. This inspires us to allocate more redundant authentication information for the more important packets to minimize the distortion of the authenticated image at the receiver. In other words, with the awareness of image content, we formulate an optimization framework, which is able to build an authentication graph yielding the best visual quality at the receiver, given a specific authentication overhead and network condition. Experimental results demonstrate that the proposed scheme achieved our design goals in that the R-D curve of an authenticated image is very close to its original one where no authentication is applied.
\end{abstract}

\section{INTRODUCTION}

Image authentication robust to packet loss can be roughly classified into content based authentication [1] and data based authentication [2]-[6]. Content based authentication involves extracting and signing the key features of the image content. Thus, it is robust against compression and network loss. The weakness of content authentication is that it sometimes cannot determine the authenticity of the received image which may be a risk on system security. Data based authentication assumes all stream packets are equally important, and tries to make every received packet verifiable. As data authentication applies one-way hash and digital signature directly to packets, it has no ambiguity in verification results and maintains the same security strength as traditional digital signature scheme.

The common approach of data authentication for a stream is to amortize one digital signature among a group of packets, which are connected as directed acyclic graph. The nodes correspond to packets and the edges correspond to hash links. For example, an edge from node $A$ to node $B$ is realized by appending $A$ 's hash to $B$. At the receiver, the lost packets are removed from the graph, and a received packet is verifiable if it has at least one path to the signature packet. Therefore, the more redundant paths a packet has to the signature packet, the higher its verification probability is. However, high redundancy also implies high authentication overhead. In other words, high verification probability and low overhead are competing goals. Two extreme examples are the simple hash chain [2] and the tree-based authentication [3]. The former has the lowest overhead and the lowest verification probability, while the latter has the highest overhead and the highest verification probability. The Augmented Chain [4], the Efficient Multi-channel Streaming Signature (EMSS) [5] and the butterfly scheme [6] are designed to balance between overhead and verification probability. However, for media streams, robustness should be measured by the visual quality instead of the verification probability, because the verified image will be ultimately perceived by humans. Furthermore, in media streams some packets are more important than others. The existing schemes do not make efficient use of the authentication overhead, as it assumes all packets are equally important.

In this paper, we propose an optimized content-aware stream authentication scheme for media streams over lossy networks. The scheme is illustrated with JPEG-2000 images, although it is also applicable to other media formats. The received image is authentic if it is exclusively decoded from authenticated packets. This definition prevents packet alteration, but allows packet loss, which is usually caused by network congestion. As such, a packet is only consumed when it is both decodable and authentic. The proposed scheme has the content information collected from the encoding process, like the distortion increments associated with packets and the inter-dependency between packets. Thus, we are able to optimally allocate the authentication overhead to individual packets in order to minimize the expected distortion of the authenticated image at the receiver. For more important packets, their hashes are replicated and appended in greater numbers to other packets, which increase their verification probability as well as the overhead. In addition, the proposed authentication 
scheme has no ambiguity in verification results and has the same security strength to traditional digital signature scheme.

The rest of the paper is organized as follows: Section 2 formulates the problem of distortion-overhead optimization. Section 3 presents the optimized content-aware authentication scheme; Section 4 evaluates and compares its performance with existing schemes through experiments; this paper is concluded in Section 5.

\section{DISTORTION-OVERHEAD OPTIMIZATION}

For the rest of the paper, we assume the distortion is additive, as used by many JPEG-2000 encoders [7]. The network is assumed to be a binary erasure channel, i.e., a datagram is either received or lost in transit. The problem of authenticating an image stream can be solved under the distortion-overhead optimization framework, which constructs an authentication graph to achieve two competing goals: minimized overhead and minimized distortion. In other words, the distortion-overhead performance should lie on the lower convex hull of the set of all achievable distortion-overhead performances.

An authentication graph is a directed acyclic graph denoted by $\langle V, G\rangle$, where $V$ is the set of nodes and $G$ is the set of directed edges in the graph. A node corresponds to a packet, and there is one and only one signature packet in $V$. A directed edge $e(i, j)$ from node $P_{i}$ to $P_{j}$ indicates the hash of $P_{i}$ is appended to $P_{j}$, where $P_{i}$ and $P_{j}$ are referred to as the source node and target node, respectively. The edge $e(i, j)$ is also referred to as a hash link that connects $P_{i}$ to $P_{j}$. The redundancy degree of $P_{i}$ is the number of edges coming out of $P_{i}$. At the receiver site, the nodes corresponding to the lost packets are removed from the graph. A received packet is verifiable if there remains a path from this packet to the signature packet. The verification probability is the probability that a received packet is verifiable. Fig. 1(a) gives an example of an authentication graph constructed at the sender side and Fig. 1(b) gives the remaining graph after packet $P_{l}$ is lost in transit.

To formulate the optimization problem, we define the vector $\pi=\left[\pi_{0}, \pi_{1}, \pi_{2}, \ldots \pi_{m}, \ldots \pi_{M-1}\right]$, where $\pi_{m}$ is the set of target nodes of the edges coming out of $P_{m}$. The redundancy degree of $P_{m}$ is $\left|\pi_{m}\right| \geq 1$. Obviously, given the set of nodes $V$, the vector $\pi$ uniquely defines the authentication graph. Suppose the total authentication overhead is $O(\pi)$ and total expected distortion of the authenticated image is $D(\pi)$, our goal is to find the optimal vector $\pi^{*}$ that minimizes the Lagrangian for given $\lambda>0$.

$$
\pi^{*}=\arg \min _{\pi}(D(\pi)+\lambda O(\pi))
$$

The overhead $O(\pi)$ is the extra bytes introduced by authentication, including hashes appended to packets and digital signature. So, $O(\pi)$ can be computed in (2), where
$S I Z_{\text {sig }}$ and $S I Z_{\text {Hash }}$ are sizes of signature and hash respectively.

$$
O(\pi)=S I Z_{\text {sig }}+\sum_{P_{m}}\left|\pi_{m}\right| S I Z_{\text {Hash }}
$$

The expected distortion $D(\pi)$ can be computed in (3), where $D_{0}$ is the distortion when no packet is consumed, $\Delta D_{m}$ is the amount of distortion reduction if $P_{m}$ is consumed, $\rho_{m}$ denotes the probability that $P_{m}$ is decodable, and $1-\varepsilon\left(\pi_{m}\right)$ denotes the probability that $P_{m}$ is verifiable with $\pi_{m}$.

$$
D(\pi)=D_{0}-\sum_{P_{m}} \Delta D_{m} \rho_{m}\left[1-\varepsilon\left(\pi_{m}\right)\right]
$$
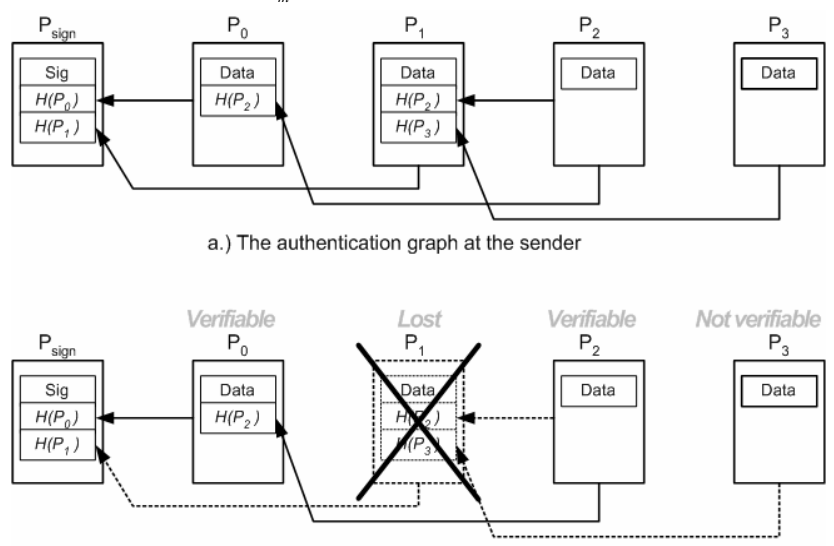

b.) The authentication graph at the receiver

Fig. 1 - Authentication graph at the sender and receiver

\section{OPTIMIZED CONTENT-AWARE AUTHENTICATION SCHEME}

In this section, we first introduce how to build an optimized authentication graph for JPEG-2000 streams, under the distortion-overhead optimization framework. We then present a simplified solution of building the authentication graph with much lower complexity.

\section{A. Distortion-overhead optimized graph}

Each JPEG-2000 packet is denoted by $P_{m}^{l}$, where the $l$ indicates the layer, and $m$ is the index of the corresponding layer- 0 packet, according to the progressive order of the codestream. The other quantities associated with $P_{m}^{l}$ are also denoted in similar way, like $\pi_{m}^{l}, \Delta D_{m}^{l}$ and $\rho_{m}^{l}$. Given a Lagrange multiplier $\lambda$, we can solve the problem by finding the vector $\pi$ that minimizes the expected Lagrangian in (4) $J(\pi)=D(\pi)+\lambda O(\pi)$

$$
=D_{0}+\lambda S I Z_{s i g}+\sum_{P_{m}^{l}}\left[\left(-\Delta D_{m}^{l} \rho_{m}^{l}\left(1-\varepsilon\left(\pi_{m}^{l}\right)\right)\right)+\lambda\left|\pi_{m}^{l}\right| S I Z_{\text {Hash }}\right]
$$

Finding the optimal variable vector $\pi$ is accomplished in two stages: the first stage is to determine $\pi_{m}^{l}$ for high layer packets $P_{m}^{l}$, i.e. $l>0$. The second stage is to determine $\pi_{m}^{0}$.

Stage 1: In JPEG-2000, the high layer packet is not decodable unless all the corresponding lower layer packets 
are decodable and authenticated, i.e. $P_{m}^{l}$ depends on $P_{m}^{k}$ for all $k$ such that $0 \leq k<l$, we say that $P_{m}^{l}$ is a descendent of $P_{m}^{k}$ and $P_{m}^{k}$ is an ancestor of $P_{m}^{l}$. In this case, one hash link connecting $P_{m}^{l}$ to one of its ancestors is sufficient, because we are interested in authenticating decodable packets. In our scheme, the target node of the only hash link from $P_{m}^{l}$ is chosen to be its immediate ancestor $P_{m}^{l-1}$, i.e. $\pi_{m}^{l}=\left\{P_{m}^{l-1}\right\}$, because choosing other ancestors are not optimal in a ratedistortion sense. Given the fixed set of hash links from all packets other than $P_{m}^{l}, \quad \pi_{m}^{l}=\left\{P_{m}^{l-1}\right\}$ will minimize the Lagrangian $J(\pi)$, as the resulting $\varepsilon\left(\pi_{m}^{l}\right)$ is equal to 0 and the redundancy degree $\left|\pi_{m}^{l}\right|$ takes the smallest value of 1 . Therefore, when $l$ is greater than 0 , the set of outgoing hash links should be $\pi_{m}^{l}=\left\{P_{m}^{l-1}\right\}$.

Stage 2: After determining $\pi_{m}^{l}$ (where $l \geq 1$ ), the probability $\rho_{m}^{l}$ can be expressed in (5), where $\phi_{m}^{i}$ denotes the probability that $P_{m}^{i}$ is received. In other words, the packet $P_{m}^{l}$ is decodable if and only if all its ancestors (namely, $P_{m}^{0}, P_{m}^{1}, \ldots, P_{m}^{l-1}$ ) and $P_{m}^{l}$ itself are received, and the corresponding layer- 0 packet, $P_{m}^{0}$, is authenticated.

$$
\rho_{m}^{l}=\left(1-\varepsilon\left(\pi_{m}^{0}\right)\right) \prod_{i=0}^{l} \phi_{m}^{i}
$$

Substitute (5) into (4), the Lagrangian $J(\pi)$ can be expressed as in (6).

$$
\begin{gathered}
J(\pi)=D_{0}+\lambda S I Z_{\text {sig }}+\sum_{P_{m}^{l}}\left[\left(-\Delta D_{m}^{l}\left(1-\varepsilon\left(\pi_{m}^{0}\right)\right) \prod_{i=0}^{l} \phi_{m}^{i}\right)+\lambda\left|\pi_{m}^{l}\right| S I Z_{\text {Hash }}\right] \\
\text { where } \quad \pi_{m}^{l}=\left\{P_{m}^{l-1}\right\} \quad \text { when } l \geq 1
\end{gathered}
$$

To ensure the authentication graph is acyclic, we mandate that for hash links whose source packet is $P_{m}^{0}$, the target packet must be $P_{n}^{0}$ where $n<m$. At this stage, for each layer-0 packet, the goal is to find the set of outgoing hash links that minimizes the Lagrangian $J(\pi)$. The straightforward method is exhaustive search, but its high computation complexity is not acceptable. A more practical approach is to use an iterative descent algorithm [8], where the objective function $J(\pi)$ is minimized one packet at a time, keeping the other packets unchanged, until convergence. For instance, let $\pi(0)=\left[\pi_{0}^{0}(0), \pi_{0}^{0}(0), \ldots\right]$ be the initial vector, and let $\pi(n)=\left[\pi_{0}^{0}(n), \pi_{0}^{0}(n), \ldots\right]$ be determined for $n=1,2, \ldots$ as follows. At iteration $n$, we select one packet, say, $P_{m}^{0}$, to find its $\pi_{m}^{0}(0)$. For $k \neq m$, let $\pi_{k}^{0}(n)=\pi_{k}^{0}(n-1)$, while for packet $P_{m}^{0}$, let

$$
\begin{gathered}
\pi_{m}^{0}(n)=\arg \min _{\pi^{\prime}} J\left(\pi_{0}^{0}(n), \pi_{1}^{0}(n), \ldots, \pi^{\prime}, \ldots\right) \\
=\arg \min _{\pi^{\prime}} \mu_{m}^{0} \varepsilon\left(\pi^{\prime}\right)+\lambda S I Z_{\text {Hash }}\left|\pi^{\prime}\right|
\end{gathered}
$$

where (7) follows from (6) with (8).

$$
\mu_{m}^{0}=\sum_{l}\left(\Delta D_{m}^{l} \prod_{i \leq l} \phi_{m}^{i}\right)
$$

At iteration $n$, it searches the set of outgoing hash links $\pi_{m}^{0}(n)$ that minimizes the Lagrangian. In the subsequent iterations, the same process is repeated. The utility value $\mu_{m}^{0}$ of the packet $P_{m}^{0}$, as expressed in (8), can be regarded as the amount by which the distortion will increase if the $P_{m}^{0}$ is not verifiable given that it is decodable. Therefore, the larger the utility $\mu_{m}^{0}$ is, the more attractive to increase the verification probability of $P_{m}^{0}$.

During the optimization process, the Lagrangian $J(\pi)$ is non-increasing at each iteration and $J(\pi) \geq 0$, so convergence is guaranteed. However, we cannot guarantee it reaches the global minimal. To increase the chance of reaching the global minimal, one alternative solution is to invoke the optimization process with different initial vectors, and choose the resulting vector that incurs the smallest Lagrangian.

\section{B. Simplified Authentication Graph}

Building the distortion-overhead optimized graph is computationally intensive. In this section, we empirically build a simplified authentication graph which requires much lower computation complexity.

For $P_{m}^{l}(l \geq 1)$, the set of outgoing hash links are exactly the same as the distortion-overhead optimized graph, i.e. $\pi_{m}^{l}=\left\{P_{m}^{l-1}\right\}$. For $P_{m}^{0}$, we compute the utility value $\mu_{m}^{0}$ using (8). After that, the packets are sorted into a list where the utility values are in descending order. The sorted list, denoted by $S L$, is then divided into $N$ segments of equal size, namely, $\operatorname{Seg}_{0}, \mathrm{Seg}_{1} \ldots, \mathrm{Seg}_{\mathrm{N}-\mathrm{l}}$. Each packet in $\mathrm{Seg}_{i}$ has $\gamma_{i}$ outgoing hash links whose target packets are randomly selected from the preceding packets in $S L$. The redundancy degree in different segments is in non-increasing order, i.e. $\gamma_{0} \geq \gamma_{1} \geq \ldots \geq \gamma_{N-1}$. Furthermore, the signature packet contains the hashes of the first $Z$ packets in $S L$.

\section{EXPERIMENTAL RESULTS}

We implemented three systems in order to evaluate the performance of the proposed scheme. The first system, WITHOUT_AUTH, is used as the upper bound for the other two systems. The second one, EMSS_AUTH, implements the EMSS scheme [5], where every packet has the same redundancy degree. The third system, CONTENT_AUTH, implements the proposed content-aware authentication 
scheme using the simplified authentication graph. Through simulation, we find that the content-aware scheme yields good performance when the parameter $N$ is 3. Further increasing $N$ does not produce substantial performance improvement, because its performance is already quite close to the upper bound when $N$ is set to 3. For both the EMSS_AUTH and CONTENT_AUTH systems, the packets are sent in the same way as in the WITHOUT_AUTH system, while the signature packet is sent multiple times to avoid loss. In the experiment, we used 8 testing images with size of 2560 pixels by 2048 pixels. For each image, we run the simulation 1000 times, and we take the average values for PSNR and the verification probability.

The network is modeled by a two-state Markov chain, where the average length of burst loss is set to 7 and the average loss probability ranges from 0.01 to 0.3 . For CONTENT_AUTH, the parameters are set as follows: $N=3, \gamma_{0}=3, \gamma_{1}=2, \gamma_{2}=1$ and $Z=6$, so the redundancy degree is 2 on average. Similarly, the EMSS_AUTH scheme uses a similar configuration, i.e. redundancy degree of 2 , and the signature packet signs the hash values of the last 6 packets. Fig. 2 gives the PSNR of the three systems. Obviously, the CONTENT_AUTH system consistently outperforms EMSS_AUTH at all network loss rates. In fact, the PSNR curve of CONTENT_AUTH is very close to the upper bound, which achieves our original design goal.

Fig. 3 compares the verification probabilities of the two authentication schemes. When the loss rate is less than 0.1, CONTENT_AUTH has slightly lower verification probability, because one third of the packets have redundancy degree of 1 . When the loss rate is large $(>0.1)$, a flat redundancy degree of 2 for all packets is not enough, which causes a dramatic decrease for EMSS_AUTH. For CONTENT_AUTH, such decrease is relatively small because one third of the packets still have redundancy degree of 3. From Fig. 2 and Fig. 3, we can draw the conclusion that CONTENT_AUTH always produce higher PNSR than EMSS_AUTH, although it sometimes may have lower verification probability. The CONTENT_AUTH scheme achieves distortion-overhead optimized performance, as the overhead is allocated in more costeffective manner.

\section{CONCLUSION}

We have proposed the optimized content-aware authentication scheme, which is able to achieve distortionoverhead optimized performance by utilizing the content and dependency information. In view that the optimization process has high computational complexity, we also propose a simplified authentication graph that requires much lower complexity to build. Through simulations, we have demonstrated that the PSNR curve of the contentaware scheme is very close to the upper bound, and it substantially outperforms existing schemes by about $3-5 \mathrm{~dB}$ on average at all loss rates. Our future work will extend the current scheme to other media formats, and take more sophisticated network conditions into consideration.

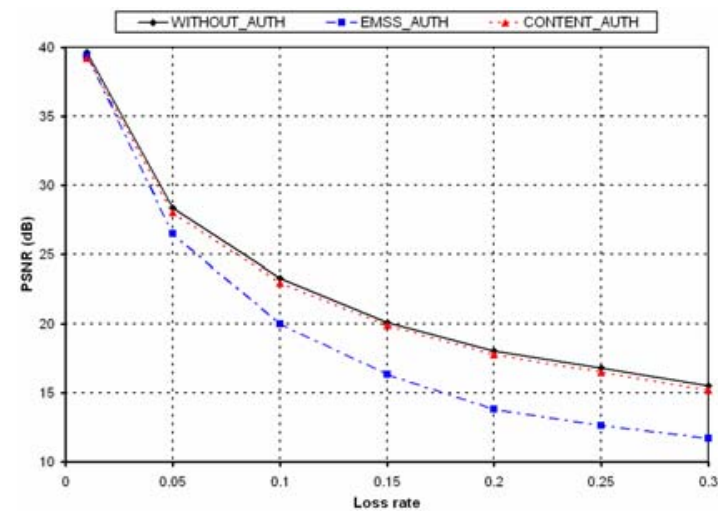

Fig. 2 - PSNR Versus Loss rates

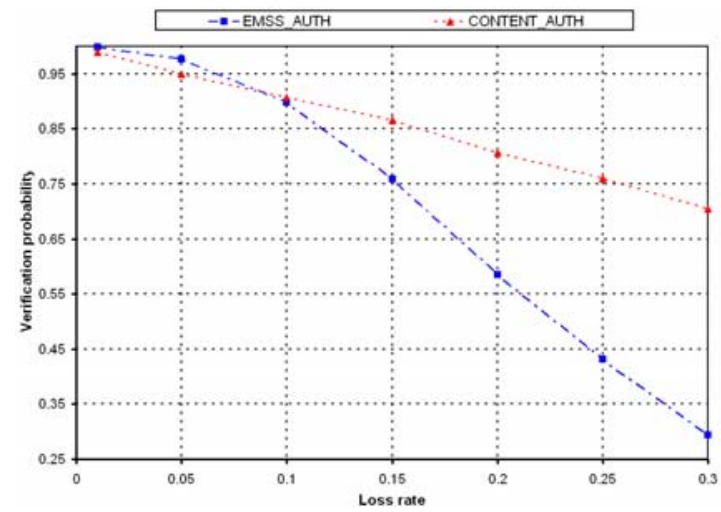

Fig. 3 - Verification probabilities Versus Loss rates

\section{REFERENCES}

Q. Sun, S. Ye, C-Y. Lin and S-F Chang, "A crypto signature scheme for image authentication over wireless channel," International Journal of Image and Graphics, Vol. 5, No. 1(2005)

[2] R. Gennaro and P. Rohatgi. "How to sign digital streams," in Advances in Cryptology - CRYPTO '97, pp. 180-197.

[3] C. K. Wong and S. Lam, "Digital Signatures for Flows and Multicasts", The University of Texas at Austin, Department of Computer Sciences, Technical Report TR-98-15. July 1998 P. Golle and N. Modadugu. "Authenticating streamed data in the presence of random packet loss," ISOC Network and Distributed System Security Symposium, 2001, pp 13--22.

[5] A. Perrig, R. Canetti, J. Tygar and D. Song. "Efficient authentication and signing of multicast streams over lossy channels," in Proc. of IEEE Symposium on Security and Privacy, 2000, pp. 56-73.

[6] Z. Zhang, Q. Sun and W-C Wong, "A Proposal of Butterflybased Stream Authentication Scheme over Lossy Networks," in Proc. of International Conf. on Multimedia \& Expo, 2005

[7] D. Taubman and M.W. Marcellin, JPEG2000: Image Compression Fundamentals, Standards and Practice, Kluwer Academic Publishers: Dordrecht, 2001, pp. 375-379

[8] R. Fletcher, Practical method of optimization, Wiley, 2nd edition 1987 